\title{
Movilización temprana en el paciente pediátrico con síndrome de Guillain-Barré. ¿Estamos iniciando de manera oportuna?
}

\section{Early mobilization in the pediatric Guillain-Barré Syndrome patient. Are we initiating in a timely manner?}

\author{
Ivonne Villanueva-Díaz', Miguel Á. Martínez-Camacho², Robert A. Jones-Baro², \\ Alberto Gómez-González ${ }^{3 *}$ y Juan S. Carrillo-Calzontzi ${ }^{3}$ \\ ${ }^{1}$ Unidad de Terapia Intensiva Pediátrica, Hospital de Especialidades del Niño y la Mujer Dr. Felipe Núñez Lara, Querétaro; ${ }^{2}$ Unidad de Cuidados \\ Intensivos Respiratorios, Hospital General de México “Dr. Eduardo Liceaga", Ciudad de México; ${ }^{3}$ Departamento de Medicina Interna, Centro Médico \\ del Noreste $N^{\circ}{ }^{\circ}$ 25, Instituto Mexicano del Seguro Social, Monterrey. México
}

\section{Resumen}

\begin{abstract}
Antecedentes: El síndrome de Guillain-Barré (SGB) puede evolucionar a fallo respiratorio agudo, requerir ventilación mecánica (VM) e ingreso a terapia intensiva. Requiere tratamiento farmacológico y fisioterapéutico. Objetivo: Establecer los días de inicio de tratamiento fisioterapéutico, número de sesiones y estancia hospitalaria. Materiales y metodología: Estudio observacional, retrospectivo, descriptivo y transversal, con revisión de expediente clínico en pacientes con SGB ingresados en la unidad de cuidados intensivos pediátricos en un periodo de seis años. El análisis estadístico incluyó medidas de tendencia central y dispersión, comparación de medianas para encontrar diferencias entre grupos. Resultados: Se registraron 12 casos, mujeres (75\%) y hombres (25\%), con respectivas edades promedio de $5.6 \pm 3.3$ años y $7.67 \pm 4.5$ años. Cuatro pacientes requirieron VM con estancia significativamente más larga (91.75 \pm 39.34 días; $p<0.05)$. La fisioterapia inició para pacientes con y sin VM, 4.21 y 3.41 días respectivamente, después del ingreso sin significación estadística ( $p$ > 0.05). Pacientes con VM recibieron más sesiones fisioterapéuticas por estancia más prolongada, sin diferencia en el número diario de terapias entre ambos grupos (VM 0.77, sin VM 0.57; $p>0.05$ ). Conclusiones: La movilización temprana por parte de fisioterapia se realizó en estos pacientes, pero con un volumen total de sesiones bajo.
\end{abstract}

Palabras clave: Movilización temprana. Fisioterapia. Pediatría. Parálisis. Autoinmune.

\section{Abstract}

Background: Guillian Barre syndrome (GBS) may evolve to acute respiratory failure, require mechanical ventilation (MV) and admission to intensive care. Requires pharmacological and physiotherapeutic treatment. Objective: To evaluate the days of start of the physiotherapeutic treatment, number of sessions and hospital stay. Materials and methodology: Retrospective descriptive cross-sectional study, with a review of the clinical record in patients with GBS admitted to the Pediatric Intensive Care Unit (PICU) over a period of 6 years. Statistical analysis included measures of central tendency and dispersion, comparison of medians to find differences between groups. Results: Twelve cases were registered, women (75\%) and men (25\%) with respective mean ages of $5.6 \pm 3.3$ years and $7.67 \pm 4.5$ years. Four patients required MV with significantly longer stay

Correspondencia:

*Alberto Gómez-González

E-mail: rehabilitacion.ag@gmail.com
Disponible en internet: 22-06-2021 Rev Hosp Jua Mex. 2021;88(2):72-75

www.revistahospitaljuarez.com 1405-9622/@ 2020 Sociedad Médico-Quirúrgica del Hospital Juárez de México, A.C. Publicado por Permanyer. Este es un artículo open access bajo la licencia CC BY-NC-ND (http://creativecommons.org/licenses/by-nc-nd/4.0/). 
(91.75 $\pm 39.34 ; p=<0.05)$. Physiotherapystarted for patients with and without MV, 4.21 days and 3.41 days, respectively, after admission without statistical significance $(p=>0.05)$. Patients with MV received more physiotherapeutic sessions for a longer stay, without difference in the daily number of therapies between both groups (with MV 0.77 , without MV 0.57; $p=>0.05$ ). Conclusions: Early mobilization (EM) by physiotherapy was performed in these patients, but with a low total session volume.

Key words: Early mobilization. Physiotherapy. Pediatrics. Paralysis. Autoimmune.

\section{Introducción}

El síndrome de Guillain-Barré (SGB) es una neuropatía de origen autoinmune ${ }^{1}$ caracterizada por debilidad simétrica ascendente, hiporreflexia o arreflexia. En México es la primera causa de parálisis flácida aguda en hospitales de primer y segundo nivel de atención ${ }^{2,3}$, y las variantes axonales son la más frecuentes a nivel nacional ${ }^{4,5}$. Mundialmente entre el año 2009 y 2010 se reportó una incidencia de 4 por 100,000 casos y Latinoamérica reporta 0.82 casos por 100,000 en menores de 15 años ${ }^{1,6}$. En el adulto, alrededor de un tercio de los pacientes requieren ventilación mecánica (VM), un $20 \%$ desarrollan déficits neurológicos severos y un $5 \%$ fallece, a pesar de recibir inmunoterapia 0 plasmaféresis. Se asocia a infecciones con Campylobacter jejuni o citomegalovirus, entre otros ${ }^{1,3,7-9}$. La inmunopatogénesis es mediante mimetismo por anticuerpos antigangliósidos en lugar de células $\mathrm{T}^{1,4,6,7}$. El pronóstico depende de la variante de SGB, las más frecuentes son: neuropatía motora axonal aguda, neuropatía sensoriomotora axonal aguda y síndrome de Miller-Fisher 1,3,8; esta última es más común en pacientes pediátricos. El tratamiento farmacológico de elección es la inmunoglobulina (lg) y en algunos casos, dependiendo de la severidad, la plasmaféresis ${ }^{9-14}$; sin embargo, se han realizado estudios comparativos entre el uso de lg vs. plasmaféresis, sin mostrar diferencias en la efectividad del tratamiento ${ }^{13,14}$. Las alteraciones residuales reportadas son dolor, limitación en la movilidad (tanto por disminución de la función como alteraciones articulares) y disminución de la respuesta cardiorrespiratoria a la realización actividades incluso años después de la recuperación de la patología, 8 ,15. También se pueden presentar complicaciones agudas que comprometen la recuperación funcional a mediano y largo plazo, con el correspondiente impacto económico y social1-3. Una de las complicaciones agudas más severas es la insuficiencia respiratoria que requiere VM e ingreso a la unidad de cuidados intensivos pediátricos (UCIP). La fisioterapia en estos pacientes es necesaria para reducir las secuelas funcionales y evitar discapacidad en edades pediátricas ${ }^{14}$ que pudieran repercutir más allá de la estancia hospitalaria, como la debilidad adquirida en la UCl, neumonía asociada a VM y úlceras por presión, entre muchas otras ${ }^{16-19}$. La movilización temprana (MT) guiada por objetivos funcionales es una de las intervenciones fisioterapéuticas más comúnmente utilizadas en pacientes críticos (Fig. 1) ${ }^{20}$. A pesar de que se recomienda la fisioterapia en etapas agudas y críticas del SGB, no se reportan estudios nacionales 0 internacionales sobre esta temática ${ }^{21-23}$.

\section{Planteamiento del problema}

La intervención y efectos de la fisioterapéutica en la $\mathrm{UCl}$ es poco estudiada en México. En el mundo no se reportan estudios de fisioterapia en SGB durante la estancia en $\mathrm{UCl}$, a pesar de conocer los efectos favorables que tiene la MT en la prevención de complicaciones derivadas de la hospitalización en otras poblaciones. Es importante mencionar que la mortalidad del SGB es baja en niños, por lo cual se debe poner especial énfasis en el estudio del desenlace funcional y en la prevención de discapacidad.

\section{Objetivos}

Establecer los días de inicio de la MT en pacientes con SGB, número de sesiones y los días de estancia hospitalaria en la UCIP del Hospital de Especialidades del Niño y la Mujer (HENM).

\section{Material y métodos}

Se trata de un estudio retrospectivo descriptivo transversal con revisión de expedientes clínicos. Se recabó la información de pacientes diagnosticados con SGB hecho por médicos en turno de forma clínica (criterios de Brighton) y laboratorios de los pacientes admitidos a la UCIP del HENM en la Ciudad de Querétaro de enero del 2014 a enero del 2020. Se calcularon los días de estancia hospitalaria, días de estancia en UCIP, días de VM, inicio de la movilización por parte del personal de fisioterapia desde el ingreso a la UCl, número de sesiones totales, así como número de sesiones por día. Se realizó estadística descriptiva y medidas de dispersión y posteriormente se dividieron los pacientes en dos 


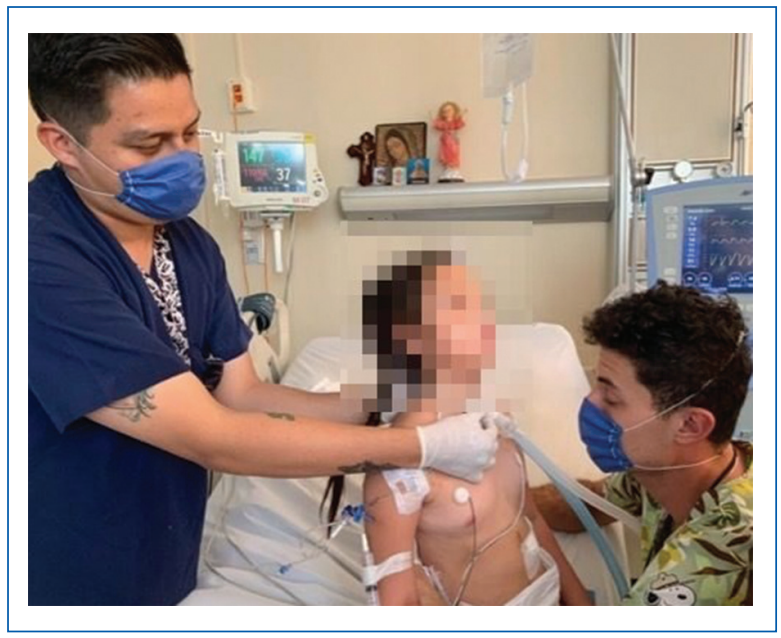

Figura 1. Sedestación a la orilla de la cama de paciente con síndrome de Guillain-Barré bajo ventilación mecánica.
Tabla 1. Características de los pacientes que requirieron ventilación mecánica vs. los que no requirieron

\begin{tabular}{|l|c|c|}
\hline Número de pacientes & $\begin{array}{c}\text { Requirió } \\
\text { ventilación } \\
\mathbf{x}( \pm)\end{array}$ & $\begin{array}{c}\text { No requirió } \\
\text { ventilación } \\
\mathbf{x}( \pm)\end{array}$ \\
\hline Edad en años cumplidos & 4 & 8 \\
\hline Días de estancia hospitalaria* & $8.25(2.98)$ & $4.88(3.52)$ \\
\hline Días de estancia en UCl* & $82.75(39.34)$ & $4.62(4.34)$ \\
\hline Inicio de movilización & $8.87(4.05)$ \\
\hline $\begin{array}{l}\text { Número de sesiones de } \\
\text { fisioterapia durante la estancia* }\end{array}$ & $63.00(32.85)$ & $2.37(2.38)$ \\
\hline Número de sesiones por día & $0.77(0.25)$ & $0.57(0.68)$ \\
\hline
\end{tabular}

*U de Mann-Whitney con valor de $p<0.05$; $x$ : media. UCI: unidad de cuidados intensivos. grupos: aquellos que requirieron de VM y los que no la requirieron. Se hizo una comparación de medianas entre grupos por medio de $U$ de Mann-Whitney debido a que se trata de una población con libre distribución, teniendo significancia estadística un grado de significación $(p)<0.05$. El análisis estadístico se realizó con el software IBM SPSS versión 21 y Microsoft Excel 2018. Los procedimientos éticos de la presente investigación toman en cuenta el Informe de Belmont, la Declaración de Helsinki, El Código de Nuremberg y La Ley General de Salud en materia de Investigación (Art. 17). Este estudio se cataloga como «Investigación sin riesgo» debido a que no se realiza ninguna intervención sobre los pacientes al momento de la investigación; se salvaguarda la confidencialidad de los datos obtenidos en la revisión de expedientes clínicos. El estudio fue aprobado por del Comité de Investigación del HENM.

\section{Resultados}

Se registraron un total de 12 casos, el $75 \%$ eran mujeres, con una edad promedio de $5.6 \pm 3.3$ años y el $25 \%$ eran hombres, con una edad promedio de 7.6 \pm 4.5 años. Solo cuatro pacientes requirieron VM, teniendo una estadía significativamente más larga dentro y fuera de la UCIP respecto a los pacientes que no requirieron VM $(82.75 \pm 39.34$ y $91.75 \pm 35.22$ vs. 4.62 \pm 4.34 y $3.41 \pm 2.79$, respectivamente; $p<0.05$ ) (Tabla 1). El servicio de fisioterapia inició MT más tarde para pacientes con VM, $4.25 \pm 1.5$ días después del ingreso en comparación con $3.41 \pm 2.7$ días para pacientes sin ventilación, pero no hubo significación estadística $(p>0.05)$. Debido a que los pacientes con VM tuvieron una estadía más prolongada, recibieron más sesiones de fisioterapia. Sin embargo, no hubo diferencia estadísticamente significativa en el número de sesiones por día con VM $(0.77 \pm 0.25)$ frente a los que no recibieron (VM $0.57 \pm 0.68)(p>0.05)$.

\section{Discusión}

Los pacientes ingresados en la UCIP por diagnóstico de SGB iniciaron MT, considerando su aplicación entre el $2 .^{\circ}$ y $5 .^{\circ}$ día del ingreso a la UCI ${ }^{18-20}$, lo que evidencia la apertura de la UCIP a la MT, como mencionan Jones, et al. ${ }^{14}$ en población pediátrica en México. Sin embargo, la media observada del número de sesiones por día es < 1 sesión/día, poniendo de manifiesto la incapacidad del servicio de fisioterapia para realizar MT en los pacientes todos los días al menos una vez al día. Esta situación se puede deber a falta de personal de fisioterapia para cubrir todos los turnos en la UCIP, situación bastante común en el país. Se observa que no hubo una diferencia significativa entre el inicio de la movilización y el número de sesiones/día en los pacientes que requirieron VM y los que no requirieron VM, lo cual indica una atención de fisioterapia homogénea a pesar de la necesidad de VM; estos pacientes tuvieron más días de estancia hospitalaria y de $\mathrm{UCl}$, y mayor número de sesiones fisioterapéuticas, lo cual se traduce en un mayor gasto de recursos hospitalarios.

Este estudio presenta limitaciones. Se trata de un estudio retrospectivo con revisión de expediente clínico, 
lo que reduce significativamente la veracidad de la información. No se describió el grado de movilidad de los pacientes ni el tipo de intervención realizada por el fisioterapeuta. Además, no se toma en cuenta el tratamiento farmacológico, lo cual puede tener implicaciones importantes en el desenlace funcional de estos pacientes. No hay estudios mexicanos que reporten los efectos de la MT en SGB, por tanto, este estudio da pauta a futuras investigaciones con la finalidad de realizar protocolos de atención en fisioterapia estructurados, guiados por objetivos funcionales y con un adecuado seguimiento en el tiempo. Esta primera información pone de manifiesto las áreas de oportunidad que tiene el servicio de terapia intensiva para el mejoramiento del proceso de rehabilitación del paciente con SGB.

\section{Conclusión}

Los pacientes ingresados en la UCIP con diagnóstico de SGB reciben MT sin importar si requirieron VM o no. Se desconoce el volumen de sesiones adecuado para modificar el pronóstico a corto, mediano y largo plazo. Se necesita realizar más estudios de seguimiento para describir el tipo de terapia con mayor efectividad para la funcionalidad en esta población, así como su impacto en la mortalidad dentro de las UCIP. Este estudio es único en su tipo y nos permite ser pionero para realizar nuevas investigaciones dentro de las diferentes patologías neurológicas mediadas por el sistema inmunitario.

\section{Agradecimientos}

Al Dr. Alejandro Rojas Macedo y al Dr. Víctor Manuel López Morales.

\section{Financiamiento}

Los autores no recibieron patrocinio para llevar a cabo este artículo.

\section{Conflicto de intereses}

Los autores declaran no tener conflicto de intereses alguno.

\section{Responsabilidades éticas}

Protección de personas y animales. Los autores declaran que para esta investigación no se han realizado experimentos en seres humanos ni en animales.
Confidencialidad de los datos. Los autores declaran que han seguido los protocolos de su centro de trabajo sobre la publicación de datos de pacientes.

Derecho a la privacidad y consentimiento informado. Los autores han obtenido el consentimiento informado de los pacientes y/o sujetos referidos en el artículo.

\section{Bibliografía}

1. Willison $\mathrm{HJ}$, Jacobs $\mathrm{BC}$, van Doorn PA. Guillain-Barré syndrome. Lancet. 2016;388(10045):717-27.

2. van den Berg B, Walgaard C, Drenthen J, Fokke C, Jacobs BC, van Doorn PA. Guillain-Barré syndrome: pathogenesis, diagnosis, treatment and prognosis. Nat Rev Neurol. 2014;10(8):469-82.

3. Carrera R, Gutiérrez S, Amaya L, Tafoya G, Aguilar S, Martínez M, et al. Síndrome de Guillain-Barré en el Hospital de Especialidades del Centro Médico Nacional Siglo XXI. Rev Med Inst Mex Seguro Soc. 2017:55(4):419-24.

4. De la O-Peña D, Robles-Figueroa M, Chávez-Peña Q, Bedolla-Barajas M. Características del síndrome de Guillain-Barré en adultos: resultados de un hospital universitario. Rev Med Inst Mex Seguro Soc. 2015;53(6):678-85.

5. Carrillo-Pérez DL, García-Ramos G, Ruano-Calderón LÁ, Sosa-Hernández JL, Méndez-Castillo JJ. Síndrome de Guillain-Barré en un hospital de referencia en México. Rev Mex Neuroci. 2012;13(1):15-21.

6. Landaverde M, Danovaro MC, Trumbo SP, Pacis CL. Guillain-Barré syndrome in children aged $<15$ years in Latin America and the Caribbean: baseline rates in the context of the influenza $A(H 1 N 1)$ pandemic. J Infect Dis. 2010;201:1-5

7. Rebolledo D, González PO, Salgado C, Síndrome de Guillain-Barré : viejos y nuevos conceptos. Med Int Mex. 2018;34(1):72-81.

8. Capasso A, Ompad DC, Vieira DL, Wilder-Smith A, Tozan Y. Incidence of Guillain-Barré Syndrome (GBS) in Latin America and the Caribbean before and during the 2015-2016 Zika virus epidemic: A systematic review and meta-analysis. PLoS Negl Trop Dis. 2019;13(8):e0007622.

9. Donofrio PD. Guillain-Barré Syndrome. Continuum. 2017;23(5):1295-309.

10. Espinosa FJ, Bergés A, Alejandra I, Dávila G, Faugier E, García JA, et al. Consenso Mexicano para la prescripción de inmunoglobulina $\mathrm{G}$ como tratamiento de reemplazo e inmunomodulación. Acta Pediatr Mex. 2018;39(2):134-71.

11. Hughes RA, Swan AV, van Doorn PA. Intravenous immunoglobulin for Guillain-Barré syndrome. Cochrane Database Syst Rev. 2014; 2014(9):CD002063.

12. Reeves HM, Winters JL. The mechanisms of action of plasma exchange. Br J Haematol. 2014;164:342-51.

13. Saad K, Mohamad IL, Abd El-Hamed MA, Tawfeek MS, Ahmed AE, Abdel Baseer KA, et al. A comparison between plasmapheresis and intravenous immunoglobulin in children with Guillain-Barré syndrome in Upper Egypt. Ther Adv Neurol Disord. 2016;9(1):3-8.

14. Jones-Baro RA, Pérez-Duarte JS, Martínez-Camacho MA, Villanueva-Díaz I, Chávez-Monjarás SM. Barreras para la movilización temprana en una unidad de cuidados intensivos pediátricos en México. Eur Sci Journal. 2019:15(21):45-58.

15. Henderson R, Lawn N, Fletcher D, McClelland R. The morbidity of Guillain-Barré syndrome admitted to the intensive care unit. Neurology. 2003;60(1):17-21.

16. Denehy L, Lanphere J, Needham DM. Ten reasons why ICU patients should be mobilized early. Intensive Care Med. 2017;43(1):86-90.

17. Martínez MA, Jones RA, Gómez A. El fisioterapeuta en la Unidad de Cuidados Intensivos ¿un profesional necesario? 2020;18(1):104-5.

18. Zhang K, Chen B, Wang M, Chen D, Hui L, Guo S, et al. The effect of early mobilization in critically ill patients: A meta-analysis. NursCrit Care. 2020;25(6):360-7.

19. Cameron S, Ball I, Cepinskas G, Choong K, Doherty TJ, Ellis CG, et al. Early mobilization in the critical care unit: $A$ review of adult and pediatric literature. J Crit Care. 2015;30(4):664-72.

20. Zhang L, Hu W, Cai Z, Liu J, Wu J, Deng Y, et al. Early mobilization of critically ill patients in the intensive care unit: A systematic review and meta-analysis. PLoS One. 2019;14(10):1-16.

21. Instituto Mexicano del Seguro Social. Diagnóstico y Tratamiento del Síndrome de Guillain Barré en el Segundo y Tercer nivel de Atención [Internet]. México: Instituto Mexicano del Seguro Social; 03/11/2016. Disponible en: http://www.imss.gob.mx/sites/all/statics/guiasclinicas/089GER.pdf

22. Durán RA, Fonseca-Chon I, Sotelo-Cruz N. Síndrome de Guillain-Barré. Experiencia con 91 niños en el Hospital Pediátrico en el Noroeste de México. Arch Neurocien. 2016;21(1):7-16.

23. Simatos Arsenault N, Vincent PO, Yu BH, Bastien R, Sweeney A. Influence of exercise on patients with Guillain-Barré syndrome: A systematic review. Physiother Can. 2016;68(4):367-76. 